\title{
Wissensaustausch beim Frühstück - „CreativeMornings" als Treffpunkt für Kultur- und Kreativschaffende in Berlin
}

\author{
Philip Müller ${ }^{1}$ Nico Schneider ${ }^{2}$ \\ Online publiziert: 16. April 2020 \\ (c) Der/die Autor(en) 2020
}

\section{Zusammenfassung}

In den letzten Jahren sind sogenannte Community Gatherings, wie Konferenzen, Messen und Branchentreffen, in den Blickpunkt von Wissenschaft, Politik und Verwaltung geraten. Diese Veranstaltungen schaffen eine institutionelle Basis für den Austausch von Wissen zwischen Akteuren, die zumeist Teil einer Community sind. Die bisherigen Studien fokussieren sich dabei vor allem auf international und national ausgerichtete Community Gatherings. Veranstaltungen mit regionaler Ausrichtung sind bisher jedoch kaum untersucht worden. Dieser Beitrag macht am Beispiel der monatlich stattfindenden Vortragsreihe von „CreativeMornings“ in Berlin deutlich, wie regionale Community Gatherings zum unternehmensübergreifenden Austausch von Wissen zwischen Kreativ- und Kulturschaffenden beitragen können. Die Ergebnisse zeigen, dass auf den Berliner CreativeMornings-Treffen ein Austausch von Wissen vor allem durch die Vorträge, die im Mittelpunkt der Veranstaltungen stehen, sowie durch die Face-to-Face-Gespräche zwischen den Teilnehmerinnen und Teilnehmern stattfindet. Das Fallbeispiel zu CreativeMornings in Berlin basiert auf der Erhebung der Nutzerprofile der Berliner CreativeMornings-Mitglieder sowie auf qualitativen Interviews mit dem Organisator und Teilnehmerinnen und Teilnehmern der Veranstaltungen.

Schlüsselwörter Community Gathering · Wissensaustausch · Kultur- und Kreativwirtschaft · Berlin · Wissensökonomie

\begin{abstract}
In recent years, so-called community gatherings such as conferences, trade fairs and branch meetings have attracted the interest of science, politics and administration. These events create an institutional basis for the exchange of knowledge between participants, most of whom are part of a specific community. Previous studies have focused primarily on internationally and nationally orientated community gatherings. However, so far, events with a regional focus have hardly been investigated. This article uses the example of the monthly lecture series of 'CreativeMornings' in Berlin, to illustrate how regional community gatherings can contribute to the inter-company exchange of knowledge between creative and cultural workers. The results show that an exchange of knowledge takes place at the CreativeMornings meetings in Berlin, mainly through talks, which are the focus of the events, but also through the face-to-face conversations between the participants. The case study on CreativeMornings in Berlin is based on a survey of the user profiles of the Berlin CreativeMornings members, as well as on qualitative interviews with the organiser and with participants in the events.
\end{abstract}

Keywords Community gathering $\cdot$ Knowledge exchange $\cdot$ Cultural and creative industries $\cdot$ Berlin $\cdot$ Knowledge economy

Dr. Philip Müller

Philip.Mueller@uos.de

Nico Schneider, M. Sc.

nico.schneider92@hotmail.de

1 Institut für Geographie, Universität Osnabrück, Seminarstr. 19a/b, 49074 Osnabrück, Deutschland

2 Hannoversche Str. 163, 37077 Göttingen, Deutschland

\section{Einleitung}

Seit einigen Jahren wird der Kultur- und Kreativwirtschaft (KKW) zunehmende Beachtung hinsichtlich der Bedeutung für das wirtschaftliche Wachstum von Regionen und Volkswirtschaften geschenkt (Comunian 2009; Boccella und Salerno 2016). Die KKW ist ein wissensintensiver Wirtschaftsbereich, der sich durch eine überdurchschnittliche Innovationsleistung im Vergleich mit anderen Branchen auszeichnet (BMWi 2014). Um wettbewerbsfähig zu blei- 
ben, sind Unternehmen in der KKW darauf angewiesen, stetig neues Wissen zu erwerben (Bathelt und Cohendet 2014). In den Blickpunkt von Wissenschaft, Politik und Verwaltung sind deshalb Treffpunkte geraten, an denen Unternehmen die Möglichkeit haben, sich zu vernetzen und neues Wissen zu akquirieren. Neben Orten der gemeinsamen Zusammenarbeit, wie „Co-Working-Spaces“, „Makerspaces“ oder „Co-Design Labs“ (Schmidt et al. 2014; Capdevila 2015; siehe Beiträge von Growe et al. und Schmidt in diesem Heft) wird vor allem sogenannten Community Gatherings, wie Konferenzen, Messen, Festivals und Branchentreffen (Klein 2011; Henn und Bathelt 2015; Comunian 2017) besondere Bedeutung beigemessen. Diese Veranstaltungen schaffen eine institutionelle Basis für den Austausch von Wissen zwischen Akteuren, die zumeist Teil einer Community sind (Henn und Bathelt 2015). Die bisherigen Studien fokussieren sich auf international und national ausgerichtete Community Gatherings. Veranstaltungen mit regionaler Ausrichtung sind bisher kaum untersucht worden. In der Praxis spielen jedoch regionale Community Gatherings als Instrument zur Förderung der KKW eine wichtige Rolle (Gnad et al. 2016).

Ziel dieses Beitrags ist es deshalb, am Beispiel der Vortragsreihe von „CreativeMornings“ (CM) in Berlin herauszuarbeiten, wie Community Gatherings als regionale Treffpunkte zum unternehmensübergreifenden Austausch von Wissen zwischen Kreativ- und Kulturschaffenden beitragen können. CM wurde 2008 durch die Schweizer Designerin Tina Roth Eisenberg in New York mit dem Ziel gegründet, regelmäßige und frei zugängliche Veranstaltungen für Kreative zu schaffen (CreativeMornings 2020). Die Veranstaltungen von $\mathrm{CM}$ finden monatlich statt und werden von Sponsoren finanziert. Derzeit werden in 211 Städten weltweit CM-Treffen organisiert, darunter auch in Berlin, Düsseldorf, Freiburg, Hamburg, Köln und München (CreativeMornings 2020, o. S.). Das Fallbeispiel zu CM in Berlin basiert auf der Erhebung der Nutzerprofile der Berliner CM-Mitglieder sowie 7 qualitativen Interviews. Die Interviews wurden mit dem Organisator der Veranstaltungen und 6 Teilnehmerinnen und Teilnehmern der Berliner CM-Treffen durchgeführt. Alle Interviews wurden transkribiert und mithilfe der qualitativen Inhaltsanalyse ausgewertet (Mayring 2000).

\section{Regionale Community Gatherings als Orte des Wissensaustauschs}

Es ist weithin anerkannt, dass sich der Prozess der Wissensentstehung in der KKW nicht auf die Rolle talentierter Personen und Organisationen beschränkt. Innovations- und Kreativitätsprozesse umfassen häufig eine Reihe weiterer Akteure, wie Kundinnen und Kunden, Auftraggeberinnen und Auftraggeber (Grabher et al. 2008) oder sogenannte Wissensgemeinschaften (Boland and Tenkasi 1995). Wissensgemeinschaften werden nach Henry und Pinch (2000, S. 194) definiert als , a group of people [...] often in separate organisations but united by a common set of norms, values and understandings, who help to define the knowledge and production trajectories of the economic sector to which they belong “. Der unternehmensübergreifende Austausch zwischen Communitymitgliedern kann dabei auf unterschiedliche Weise stattfinden, etwa in Onlineforen (z.B. Grabher und Ibert 2014), an Orten der gemeinschaftlichen Zusammenarbeit (z. B. Schmidt et al. 2014) und auf Community Gatherings (z. B. Comunian 2017).

Community Gatherings, wie Konferenzen, Messen und Branchentreffen, schaffen Gelegenheiten, an denen Mitglieder einer Wissensgemeinschaft in größerer Zahl aufeinandertreffen können. Bisherige Arbeiten, die sich vor allem auf international ausgerichtete Veranstaltungen fokussieren, zeigen, dass diese eine wichtige Rolle für den Austausch von Wissen innerhalb von Communities spielen. Zum einen stellen sie eine Möglichkeit zum direkten Wissensaustausch zwischen Communitymitgliedern dar. Die Teilnehmerinnen und Teilnehmer der Veranstaltungen haben die Möglichkeit, sich über ihre Erfahrungen sowie aktuelle Trends, zukünftige Produkte, Dienstleistungen und Innovationen auszutauschen (Bathelt und Schuldt 2008). Aufgrund ihrer Zugehörigkeit zu einer bestimmten Wissensgemeinschaft ist es den Teilnehmerinnen und Teilnehmern zumeist möglich, Wissen effizient zu teilen und zu interpretieren (Henn und Bathelt 2015). Regionale Community Gatherings können dabei als temporäre Orte des lokalen Rauschens („local buzz") verstanden werden, in denen Neuigkeiten, strategische Informationen, Erfahrungswissen, Gerüchte und Empfehlungen verbreitet werden (Bathelt et al. 2004).

Zum anderen bieten Community Gatherings Gelegenheiten zur Pflege bestehender sowie zum Aufbau neuer Kontakte, die in Zukunft nutzbar gemacht werden können. Diese Funktion wird besonders für internationale Community Gatherings hervorgehoben (Bathelt und Schuldt 2008; Henn und Bathelt 2015). Doch auch auf regionaler Ebene kann Community Gatherings dahingehend eine wichtige Bedeutung zukommen (z.B. Müller und König 2018). So sind gerade in Metropolregionen die Beziehungen zwischen Mitgliedern einer Wissensgemeinschaft nicht automatisch in großer Anzahl vorhanden (Bathelt und Cohendet 2014). Somit können regionale Community Gatherings verhindern, dass Beziehungen zwischen einzelnen Akteuren in der Wissensgemeinschaft zu eng und exklusiv werden. Dies ist von wesentlicher Bedeutung für die Innovationsfähigkeit und die Kreativität von Akteuren der KKW. Denn es sind vor allem die sogenannten schwachen Verbindungen (,weak ties"), die Akteuren den Zugang zu neuem, abweichendem Wissen bereitstellen (Granovetter 1973). 


\section{Fallbeispiel: CreativeMornings in Berlin}

\section{Organisation und Ziele der Veranstaltungen}

Die Veranstaltungen von CM in Berlin finden seit August 2011 statt, in der Regel an einem Freitagmorgen im Monat. Ziel von CM ist es, den Teilnehmerinnen und Teilnehmern einen Treffpunkt zum Austausch mit anderen Kreativen bei einem gemeinsamen Frühstück zu bieten (Interview 1, Organisator). Für die CM-Veranstaltungen wird kein Eintritt verlangt. Einzige Voraussetzung zur Teilnahme ist die Registrierung auf der Webseite von CM sowie die Anmeldung für das jeweilige Treffen (CreativeMornings 2020). Je nach Größe des Veranstaltungsorts variieren die Teilnehmerzahlen, wobei durchschnittlich 100 Teilnehmerinnen und Teilnehmer an den Treffen in Berlin partizipieren (Interview 1, Organisator). Die Treffen finden jeweils an wechselnden Veranstaltungsorten statt. Dadurch soll den Teilnehmerinnen und Teilnehmern aus verschiedenen Bezirken Berlins ein leichterer Zugang zu den Treffen ermöglicht werden. Gleichzeitig soll dadurch die Bekanntheit von CM in Berlin erweitert werden (Interview 1, Organisator). Im Zentrum der Veranstaltungen steht jeweils ein Vortrag (siehe Abb. 1), der circa $20 \mathrm{~min}$ dauert. Vor und nach dem Vortrag haben die Teilnehmerinnen und Teilnehmer die Gelegenheit, sich untereinander auszutauschen (CreativeMornings 2020).

\section{Charakteristik der Teilnehmerinnen und Teilnehmer}

Auf der Webseite von CM Berlin sind 1890 Communitymitglieder registriert, von denen bereits $78,5 \%$ mindestens einmal physisch an einem CM-Treffen teilgenommen haben (Stand 2017). Der überwiegende Teil der Mitglieder hat bislang nur einmal (knapp $40 \%$ ) bzw. maximal fünfmal (ca. 26\%) an CM-Treffen teilgenommen. Lediglich 1,3\% der Mitglieder hat an 20 oder mehr Treffen in Berlin partizi-

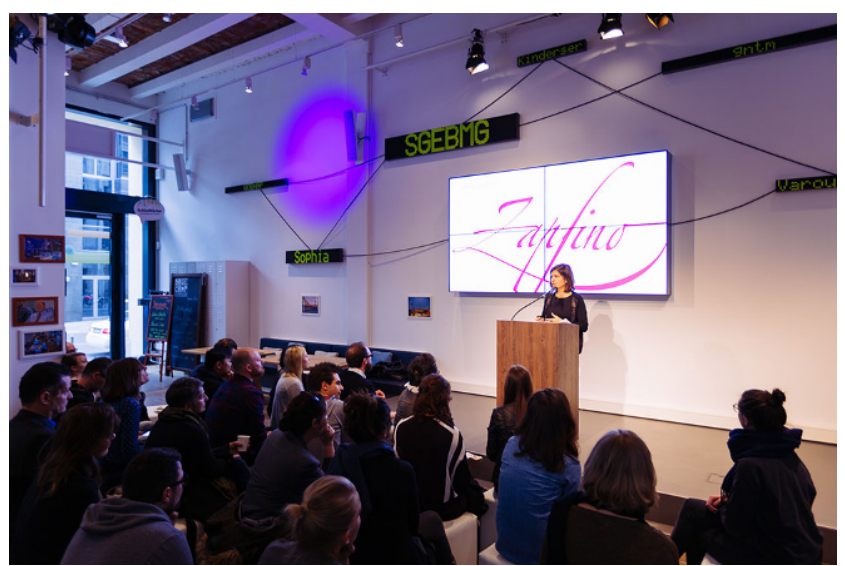

Abb. 1 Vortrag beim Berliner CreativeMornings-Treffen am 17. April 2015 (Foto: Norman Posselt) piert. Hinsichtlich der Zugehörigkeit der Mitglieder zu den Teilbranchen der KKW lässt sich ein Schwerpunkt in der Designwirtschaft sowie der Software- und Gamesindustrie feststellen. Mit knapp 700 (37,2\%) zur Designwirtschaft zugeordneten Mitgliedern stellt diese Teilbranche die mit Abstand präsenteste innerhalb von $\mathrm{CM}$ in Berlin dar. Der Software- und Gamesindustrie sind 18,4\% der Mitglieder zuzuordnen. Auffällig ist auch der mit 18,6\% relativ hohe Anteil derjenigen, die in keiner der Teilbranchen der KKW tätig sind. Hierunter fallen bspw. Businesscoaches, Studierende oder Headhunter (eigene Erhebung 2017).

\section{Austausch von Wissen}

Die CM-Treffen in Berlin bieten den Teilnehmerinnen und Teilnehmern 2 wesentliche Möglichkeiten zum direkten Austausch und Erwerb von Wissen: durch die Vorträge und anschließenden Fragerunden sowie durch die Face-toFace-Gespräche zwischen den Teilnehmerinnen und Teilnehmern. Die Vorträge werden von Referentinnen und Referenten gehalten, die in den unterschiedlichsten Bereichen sowohl innerhalb als auch außerhalb der KKW tätig sind. Dadurch bieten die Vorträge den Teilnehmerinnen und Teilnehmern zum einen detaillierte Einblicke in die Arbeitsweisen sowie Problemlösungsstrategien anderer Kreativer. „What I see as the most value which I get from the talks is just to see how different people are working. How differently they are trying to solve this issue of creativity or how to be creative. And this helps me the most I would say. Just to realize you can go this way or that way and it always works" (Interview 6, Teilnehmer). Zum anderen bieten sie den Teilnehmerinnen und Teilnehmern einen Perspektivwechsel und Eindrücke aus anderen Bereichen und Branchen. „Bei mir ist es immer so, dass es für mich spannender ist, jemanden sprechen zu hören, der nicht Designer ist, oder nicht in dem gleichen Bereich ist wie ich. $D a$, glaube ich, bietet sich CreativeMornings ganz gut an dafür" (Interview 4, Teilnehmerin). Die Vortragsinhalte haben dabei eine große Bandbreite, die von Kreativität und Design bis zu Empathie und Überwachung reicht. Viele der Befragten gaben an, dass die Vorträge sie auf neue Ideen gebracht haben und als Quelle der Inspiration dienen. Eine wichtige Rolle beim Wissenserwerb durch die Vorträge spielen auch die Fragerunden, in der die Teilnehmerinnen und Teilnehmer Nachfragen stellen und die Vortragsinhalte vertiefen können (siehe Abb. 2). Nach dem Vortrag und der Fragerunde haben die Teilnehmerinnen und Teilnehmer zudem die Möglichkeit, mit den Referentinnen und Referenten ins Gespräch zu kommen.

Die meisten Befragten nutzen die CM-Treffen nicht nur, um sich den Vortrag anzuhören, sondern auch um sich mit anderen Teilnehmerinnen und Teilnehmern auszutauschen (siehe Abb. 3). Die Auswahl der Gesprächspartnerinnen und 


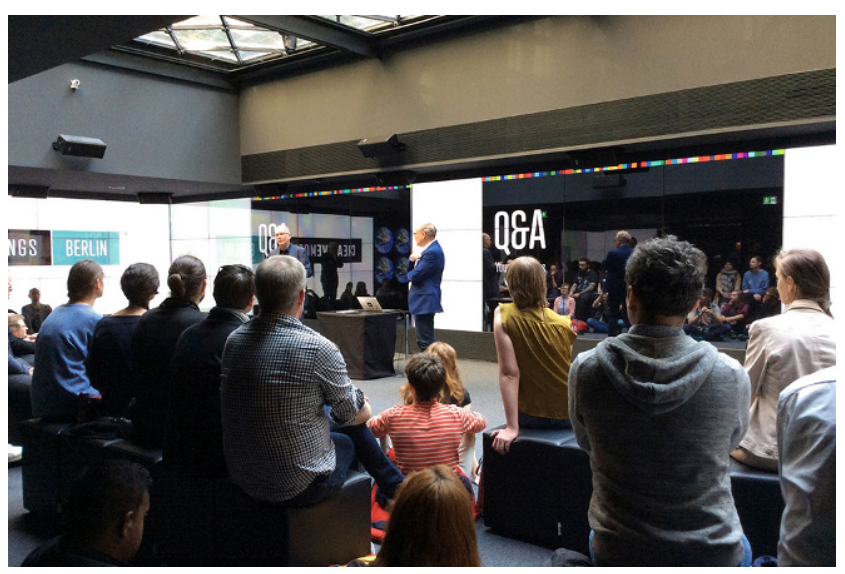

Abb. 2 Fragerunde beim Berliner CreativeMornings-Treffen am 28. April 2017 (Foto: Nico Schneider)

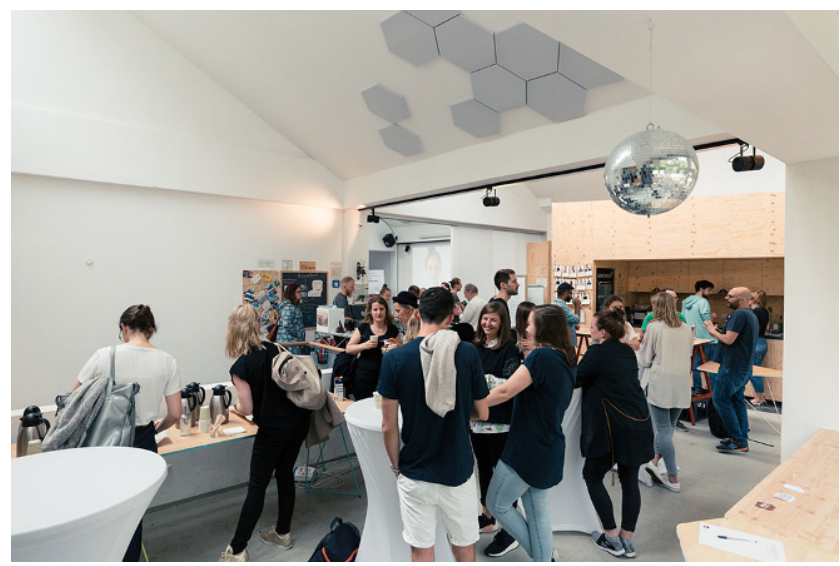

Abb. 3 Austausch zwischen den Teilnehmerinnen und Teilnehmern beim Berliner CreativeMornings-Treffen am 7. Juli 2017 (Foto: Norman Posselt)

Gesprächspartner findet in den meisten Fällen nicht zielgerichtet, sondern eher zufällig statt. Nur wenige Befragte nutzten im Vorfeld der Treffen die Möglichkeit, die Teilnehmerliste über die Webseite einzusehen, um so vorab einen Überblick über die anderen Teilnehmerinnen und Teilnehmer zu bekommen und mögliche, interessante Gesprächspartnerinnen und Gesprächspartner auszumachen. In den Gesprächen zwischen den Anwesenden werden neben den Inhalten aus dem Vortrag häufig auch andere Themen diskutiert. So nutzen die Befragten die Gespräche vor allem dazu, Erfahrungswissen zu teilen, bspw. indem Empfehlungen und Tipps für Projekte ausgetauscht werden: „For example, when I was running a Kickstarter campaign I hardly knew anyone who did something similar [...] then we exchanged some experiences how it works and how to make it better" (Interview 6, Teilnehmer). Neben dem Erfahrungswissen tauschen die Teilnehmerinnen und Teilnehmer auch Branchenwissen aus: , ,[...] was geht gerade in der Industrie $a b$, worüber spricht man, was sind die aktuellen Tendenzen und
Trends“ (Interview 3, Teilnehmer). Damit stärken die CMVeranstaltungen die Zirkulation von Wissen und Informationen innerhalb der Berliner KKW. Sie lassen sich somit als temporäre Orte des lokalen Rauschens charakterisieren (Bathelt et al. 2004).

Die CM-Treffen werden von den Befragten zudem zur Aufrechterhaltung und zum Ausbau ihres persönlichen Netzwerks genutzt. So verabreden sich einige der Befragten mit Bekannten oder ehemaligen Kolleginnen und Kollegen zu den Veranstaltungen, um bereits vorhandene Beziehungen zu pflegen. Die meisten Befragten sehen die CM-Treffen aber vor allem als Gelegenheit, um mit vorher unbekannten Teilnehmerinnen und Teilnehmern ins Gespräch zu kommen und somit eventuell neue Kontakte zu knüpfen. Das Organisationsteam der Berliner CMTreffen unterstützt die Teilnehmerinnen und Teilnehmer dabei, neue Austausch- und Kooperationspartner zu finden. Dies geschieht vor allem über sogenannte 30-SekundenPitches, in denen Teilnehmerinnen und Teilnehmer auf der Bühne über ihre Ideen und derzeitigen Projekte berichten (Interview 1, Organisator). Ein Teilnehmer beschreibt diese Methode wie folgt: „[I]n den 30-Second Pitches geht es halt darum, kleinere Ideen zu präsentieren. Die reichen halt nicht für einen Talk [...]. Aber die sind wie eine kleine Suchanzeige, wie eine kleine Annonce, die man schaltet, mit der man dann hoffentlich die richtigen Leute erreicht" (Interview 2, Teilnehmer). Zumeist geht der Austausch zwischen den Teilnehmerinnen und Teilnehmern nicht über die CM-Veranstaltungen hinaus. Durch die Treffen entstehen vielmehr potenzielle Kontakte, die in Zukunft relevant werden könnten. Ein Interviewpartner beschreibt dies wie folgt: „Da sind bei mir dann auch in der Tat einige berufliche Gelegenheiten entstanden. Jetzt nicht so konkret direkt, dass man sich auf einem CreativeMornings trifft und dann gleich zusammenarbeitet, aber dass man sich von da her irgendwie kennt. Und dann war es bei uns zum Beispiel der Fall, dass wir ein Logo brauchten. Dann ist mir eingefallen, dass ich da diese Letterdesignerin kenne aus Berlin, die war auch öfter bei CreativeMornings. Dann habe ich sie angeschrieben und dann hat sich daraus was ergeben " (Interview 3, Teilnehmer). Das Zitat verdeutlicht, dass durch die CM-Treffen neue schwache Verbindungen geschaffen werden (Granovetter 1973), wodurch die wirtschaftliche Zusammenarbeit zwischen Kultur- und Kreativschaffenden in Berlin gestärkt wird.

\section{Fazit}

Der vorliegende Beitrag zeigt am Beispiel der monatlich stattfindenden Vortragsreihe von CreativeMornings (CM) in Berlin, wie Community Gatherings als regionale Treffpunkte zum unternehmensübergreifenden Austausch von Wissen 
zwischen Kreativ- und Kulturschaffenden beitragen können. Auf den Berliner CM-Treffen findet ein Austausch von Wissen vor allem durch die Vorträge, die im Mittelpunkt der Veranstaltungen stehen, sowie durch die Face-toFace-Gespräche zwischen den Teilnehmerinnen und Teilnehmern statt. Die Vorträge werden von Referentinnen und Referenten gehalten, die in unterschiedlichsten Bereichen innerhalb und auch außerhalb der Kultur- und Kreativwirtschaft (KKW) tätig sind. Dadurch werden Wissen und Informationen $\mathrm{zu}$ einer großen Bandbreite von Themen vermittelt. Für die Teilnehmerinnen und Teilnehmer sind die Vorträge dabei Inspirationsquelle und Anstoß für neue Ideen. Die Gespräche zwischen den Teilnehmerinnen und Teilnehmern werden genutzt, um sich sowohl über die Inhalte des Vortrags auszutauschen, als auch um Erfahrungs- und Branchenwissen zu teilen. Somit stellen CM-Treffen temporäre Orte des lokalen Rauschens (,local buzz“) dar (Bathelt et al. 2004). Die Veranstaltungen erhöhen außerdem die Vernetzung zwischen Kultur- und Kreativschaffenden in Berlin.

Die Ergebnisse verdeutlichen, dass die KKW in Städten und Regionen von niedrigschwelligen Veranstaltungsformaten wie CreativeMornings profitieren kann. Zur Umsetzung dieser Treffen müssen nur wenige Voraussetzungen erfüllt sein. Es muss Personen geben, die die Organisation der Treffen übernehmen. Neben geeigneten Räumlichkeiten ist zudem eine kritische Masse an möglichen Teilnehmerinnen und Teilnehmern erforderlich. In einigen Regionen erfahren derartige Veranstaltungen eine öffentliche Förderung, wie sich u. a. in Hamburg und München zeigt. So richtet die Hamburg Kreativ Gesellschaft regelmäßig einen „Gamecity Treff“ aus, um Akteure aus der regionalen Gamesbranche zusammenzubringen (Hamburg Kreativ Gesellschaft 2020). In München veranstaltet das städtische Kompetenzteam Kultur- und Kreativwirtschaft regelmäßig stattfindende regionale „KuK-Connect“"-Treffen mit wechselnden inhaltlichen Schwerpunkten (Kompetenzteam Kultur- und Kreativwirtschaft 2020).

Funding Open Access funding provided by Projekt DEAL.

Open Access Dieser Artikel wird unter der Creative Commons Namensnennung 4.0 International Lizenz veröffentlicht, welche die Nutzung, Vervielfältigung, Bearbeitung, Verbreitung und Wiedergabe in jeglichem Medium und Format erlaubt, sofern Sie den/die ursprünglichen Autor(en) und die Quelle ordnungsgemäß nennen, einen Link zur Creative Commons Lizenz beifügen und angeben, ob Änderungen vorgenommen wurden.

Die in diesem Artikel enthaltenen Bilder und sonstiges Drittmaterial unterliegen ebenfalls der genannten Creative Commons Lizenz, sofern sich aus der Abbildungslegende nichts anderes ergibt. Sofern das betreffende Material nicht unter der genannten Creative Commons Lizenz steht und die betreffende Handlung nicht nach gesetzlichen Vorschriften erlaubt ist, ist für die oben aufgeführten Weiterverwendungen des Materials die Einwilligung des jeweiligen Rechteinhabers einzuholen.
Weitere Details zur Lizenz entnehmen Sie bitte der Lizenzinformation auf http://creativecommons.org/licenses/by/4.0/deed.de.

\section{Literatur}

Bathelt H, Cohendet P (2014) The creation of knowledge: local building, global accessing and economic development-toward an agenda. J Econ Geogr 14(5):869-882

Bathelt H, Schuldt N (2008) Between luminaires and meat grinders: International trade fairs as temporary clusters. Reg Stud 42(6):853-868

Bathelt H, Malmberg A, Maskell P (2004) Clusters and knowledge: local buzz, global pipelines and the process of knowledge creation. Prog Hum Geogr 28(1):31-56

Boccella N, Salerno I (2016) Creative economy, cultural industries and local development. Procedia Soc Behav Sci 223:291-296

Boland RJ, Tenkasi RV (1995) Perspective making and perspective taking in communities of knowing. Organ Sci 6:350-372

Bundesministerium für Wirtschaft und Technologie (2014) Monitoring zu ausgewählten wirtschaftlichen Eckdaten der Kultur- und Kreativwirtschaft 2014. https://www.kultur-kreativ-wirtschaft. $\mathrm{de} / \mathrm{KuK} /$ Redaktion/PDF/monitoring-wirtschaftliche-eckdatenkuk-2014, property=pdf, bereich=kuk, sprache=de, rwb=true.pdf. Zugegriffen: 12. Mai 2017

Capdevila I (2015) Co-working spaces and the localised dynamics of innovation in Barcelona. Int J Innov Mgt 19(3):1-25

Comunian R (2009) Questioning creative work as driver of economic development: the case of Newcastle-Gateshead. Creat Ind J 2(1):57-71

Comunian R (2017) Temporary clusters and communities of practice in the creative economy: Festivals as temporary knowledge networks. Space Cult 20(3):329-343

CreativeMornings (2020) CreativeMornings. https://creativemornings. com. Zugegriffen: 24. Febr. 2020

Gnad F, Ebert R, Kunzmann KR (2016) Kultur-und Kreativwirtschaft in Stadt und Region. Branchen-Orte-Netze. Kohlhammer, Stuttgart

Grabher G, Ibert O (2014) Distance as asset? Knowledge collaboration in hybrid virtual communities. J Econ Geogr 14(1):97-123

Grabher G, Ibert O, Flohr S (2008) The neglected king: The customer in the new knowledge ecology of innovation. Econ Geog 84(3):253-280

Granovetter M (1973) The strength of weak ties. Am J Sociol 78(6): 1360-1380

Hamburg Kreativ Gesellschaft (2020) Gamecity: Hamburg. Netzwerk der Gamesbranche. https://kreativgesellschaft.org/innovation/ gamecity-hamburg. Zugegriffen: 10. März 2020

Henn S, Bathelt H (2015) Knowledge generation and field reproduction in temporary clusters and the role of business conferences. Geoforum 58:104-113

Henry N, Pinch S (2000) Spatialising knowledge: placing the knowledge community of Motor Sport Valley. Geoforum 31(2):191-208

Klein RR (2011) Where music and knowledge meet: a comparison of temporary events in Los Angeles and Columbus, Ohio. Area 43(3):320-326

Kompetenzteam Kultur- und Kreativwirtschaft (2020) Kompetenzteam Kultur- und Kreativwirtschaft. Unterstützung für Münchens Kreativschaffende. https://www.muenchen.de/rathaus/wirtschaft/ branchen/kreativwirtschaft/kompetenzteam.html. Zugegriffen: 13. März 2020

Mayring P (2000) Qualitative content analysis. Forum Qual Soc Res. https://doi.org/10.17169/fqs-1.2.1089

Müller P, König T (2018) How do online community platforms and associated offline meetings support highly skilled (re-)migration? The case of the Rückkehrerstammtisch in Istanbul. Erde 149(4):199-213 
Schmidt S, Brinks V, Brinkhoff S (2014) Innovation and creativity labs in Berlin. Z Wirtschgeogr 58(1):232-247

Dr. Philip Müller Jg. 1987. Studium der Geographie, Wirtschaftswissenschaften, Soziologie und Politikwissenschaften an der TU Dresden, Universität Aalborg und der Philipps-Universität Marburg. Seit 2014 als wissenschaftlicher Mitarbeiter in der Arbeitsgruppe Humangeographie mit wirtschaftsgeographischen Schwerpunkt am Institut für Geographie der Universität Osnabrück beschäftigt. Seine Forschungsschwerpunkte sind die internationale Mobilität hochqualifizierter Arbeitskräfte, globale Unternehmensverflechtungen und die Reorganisation von Wertschöpfungsprozessen.
Nico Schneider Jg. 1992. Studium der Geographie an der GeorgAugust-Universität Göttingen und der Universität Osnabrück. Studienschwerpunkt waren insbesondere wirtschaftsgeographische Themen wie die geographische Handelsforschung sowie Investitionsbeziehungen. Seine Masterarbeit schrieb er 2017 über Wissensaustausch in temporären Settings am Beispiel von CreativeMornings in Berlin und Utrecht. Nico Schneider arbeitet seit Anfang 2018 bei Burger King Deutschland. Zuletzt war er als Manager Expansion and Real Estate tätig. 